Z Herz-Thorax- Gefäßchir 2011

[Suppl 1] 25:3-5

DOI 10.1007/s00398-011-0830-5

Online publiziert: 14. Mai 2011

(c) Springer-Verlag 2011

\author{
H. H. Scheld \\ Klinik und Poliklinik für Thorax-, Herz- und Gefäßchirurgie, Universität Münster
}

\title{
Das interdisziplinäre Münsteraner Modell
}

Wort Couch ist. So sehr bestimmt heute eine popularisierte Form der Psychoanalyse das Verständnis der Allgemeinheit von Psychologie. Nun könnte man natürlich sagen: Es ist doch unbedeutend, was Ärzte über die Psychologie denken. Aber dem ist nicht so. Wie bei anderen Berufen auch - man denke an Spitzenmanager oder Politiker - hat das Image eines Berufsstands oft bedeutsame Folgen. Im Fall der Psychologen verringert es beispielsweise die Bereitschaft von Menschen mit psychischen Problemen, sich frühzeitig in therapeutische Behandlung zu begeben.

\section{(D) Die klinische Psychologie ist für die gesamte Herzchirurgie sehr wichtig.}

Kommen wir aber nun zu den Erfahrungen, die ich in den vergangenen 35 Jahren als Herzchirurg mit der Psychologie gemacht habe. Ich könnte jetzt chirurgisch kurz und knapp sagen: Meine Erfahrungen sind sehr gut. Ich denke jedoch, das würde der Sache nicht gerecht werden und deshalb werde ich es ein wenig ausführen. Auslöser des Interesses an der Psychologie war die Tatsache, dass ich zu Beginn der 7oer Jahre immer wieder postoperative Durchgangssyndrome erleben musste, denen wir Chirurgen zum größten Teil hilflos gegenüberstanden. Nach einigen Jahre hatte ich aber festgestellt, dass man bei den meisten Patienten präoperativ vorhersagen konnte, ob und welches Ausmaß das postoperative Psychosyndrom haben würde. Ich habe daher schon zu meiner Gießener Zeit mit einer Arbeitsgruppe aus Psychosomatikern und Psychologen sehr aufwendige
Untersuchungen an unseren herzchirurgisch operierten Patienten durchgeführt. Hierbei wurden die Patienten bis über das erste Jahr nach der Operation betrachtet. Während dieser Zeit habe ich erheblich vom Umgang und der Arbeitsweise unserer Psychologen gelernt.

Darüber hinaus war ein weiterer persönlicher Bezug zur medizinischen Psychologie eng mit dem Beginn unserer Transplantationsprogramme, zunächst an der Justus-Liebig-Universität Gießen und später an der Westfälischen WilhelmsUniversität in Münster, verbunden. Zur damaligen Zeit verfügte noch kein einziges Herzzentrum in Deutschland über eine solche Position. Entsprechend stieß ich in Münster auf Ablehnung, Unverständnis und große Skepsis, als ich diesen damals noch ungewöhnlichen Wunsch vortrug. So mancher machte sich lustig über meine Vorstellung, dass zur Transplantationsmedizin unbedingt eine psychologische Betreuung gehört. Unser damaliger Verwaltungsdirektor Gotthardt aber bewies Weitblick und so konnte ich in Zusammenarbeit mit dem Arbeitsamt über eine arbeitsplatzbildende Maßnahme eine Psychologenstelle für meine Klinik erwirken, die uns heute unentbehrlich geworden ist und wofür ich immer noch dankbar bin. Mein Ziel Anfang der 9oer Jahre war die Integration. Die Reaktionen aber waren so heftig, als würde ich hier eine stillschweigende feindliche Übernahme eines Faches durch ein anderes vorbereiten. Inzwischen ist die damals eingestellte Stelleninhaberin habilitiert und auf ihrem Spezialgebiet der Transplantationspsychologie aufgrund ihrer praktischen und wissenschaftlichen Tätigkeit bekannt. 
Die anfänglich skeptischen Stimmen am Universitätsklinikum Münster sind verstummt.

Die klinische Psychologie ist aus meiner Sicht ein sehr wichtiger Faktor - nicht nur in der Transplantationsmedizin, sondern auf dem gesamten Gebiet der Herzchirurgie. Eine Herzoperation ist für uns Chirurgen eine Routinemaßnahme zur Besserung der oft gravierenden Beschwerden und der reduzierten Lebensqualität unserer Patienten. Für den einzelnen Patienten jedoch ist sie eine bedrohliche Situation. Das Herz hat nun einmal aufgrund seiner zentralen Stellung im Körper und seiner emotionalen Bedeutung einen besonderen Rang unter den Organen und häufig zwingen Herzoperationen den Betroffenen zur gedanklichen Auseinandersetzung mit dem Tod. Auch die emotionale Überfrachtung des Herzens bleibt nicht ohne Auswirkungen auf die Psyche. Alle Herzpatienten empfinden Ängste um ihr Herz, oftmals sogar Todesängste. Mir war auch schon aus einer Arbeit von Richard Blacher (1989) bekannt, dass sich die Patienten unabhängig vom tatsächlichen Risiko, das bei 2-4\% liegt - eine Chance von 50:50 geben, den Eingriff zu überleben. Aus diesem Grund brauchen die Patienten schon vor dem Eingriff Unterstützung und Zuspruch und es verwundert nicht, dass viele nach dem Erhalten des Einbestellungsbriefs zur Herzoperation mittlerweile auch einen Termin mit unserer Psychologin vereinbaren.

Es ist aber leider auch eine traurige Realität, dass während eines Klinikaufenthalts nicht immer alles so verläuft, wie wir Chirurgen es uns wünschen. In Einzelfällen kann es bei den Herzoperationen zu Ereignissen vor, während und nach der Operation kommen, die den Heilungsprozess verzögern oder gar verhindern. Die anschließend notwendigen Gespräche mit den Angehörigen erfordern aktives Zuhören und konkretes Eingehen auf die bestehenden Ängste. Gelernt habe ich zudem, dass die Bewältigungsmechanismen der einzelnen Familien und Patienten von dem lebensgeschichtlichen Erfahrungshintergrund und den situativen Bedingungen des Klinikumsumfelds abhängig sind. So konnte ich in den letzten Jahren erleben, wie wichtig es ist, in unserem sehr hektischen medizinischen All- tag die Möglichkeit zu haben, die Angehörigen mit einer Fachkraft zusammenzubringen, die ihnen mit Trost und Rat, aber auch mit wissenschaftsbasierter psychologischer Therapie zur Seite steht. Hinzu kommt, dass wir als Herzchirurgen ruhig zugeben dürfen, dass es für uns nicht leicht zu bestimmen ist, welche Reaktionen und Verhaltensweisen einer psychologischen Betreuung und welche einer längeren psychotherapeutischen Behandlung bedürfen.

Immer wieder können wir erleben, dass Familie und Freunde während des Krankenhausaufenthalts zwar eine wichtige Stütze sind, sie können aber nicht alle Probleme und Unsicherheiten auffangen, da sie selbst oft sehr ängstlich sind. Deshalb ist auch hier eine psychologische Betreuung sinnvoll und immer wieder haben mir die Rückmeldungen gezeigt, wie entlastend es für die Angehörigen ist, mit unserer Psychologin Ängste und Sorgen besprechen zu können, ohne die Patienten selbst damit zu belasten.

\section{(2) Familie und Freunde können nicht alle Probleme und Unsicherheiten auffangen.}

Heute werden Herztransplantationen als Standardverfahren durchgeführt. Kaum ein Betroffener würde jedoch in Frage stellen, dass eine Herztransplantation neben dem großen medizinischen Eingriff auch ein großer Eingriff in den Alltag und das Leben des Betroffenen und der Angehörigen ist. Die zahlreichen Situationen, die für die Patienten und ihre Angehörigen psychische Belastungen bedeuten, sprechen hier eine eindeutige Sprache. Wir haben in Münster seit 1990 (also 7 Jahre vor Inkrafttreten des neuen Transplantationsgesetzes) ein interdisziplinäres Modell integriert und sehr gute Erfahrungen damit gesammelt. In unserem interdisziplinären Ansatz ist die psychologische Betreuung ein fester Bestandteil und beginnt bereits vor der Listung mit einer standardisierten psychosozialen Evaluation und nicht erst als Ultima ratio. Für die Patienten ist sie ein selbstverständlicher Bestandteil unseres Betreuungsangebots. Damit gelingt es, die Annahmeschwelle deutlich zu senken. Hinzu kommt die Notwendigkeit, die psychologische Betreuung nicht als Feuer- wehreinsatz zu sehen, da sie von Beginn an durch Studien unterstützt wird, die zeigen, dass sich spätere Belastungen oft aus früheren vorhersagen lassen.

Transplantationen sind komplexe Operationen mit komplizierten Behandlungsstrategien. Fast ebenso komplex wie die Operation ist auch die psychische Auseinandersetzung des Patienten mit dem Eingriff. Wir alle haben doch schon erlebt, dass die betroffenen Patienten nicht selten eine Achterbahn der Gefühle durchlaufen. Sie berichten, dass ihnen durch dieses Gesprächsangebot viel Ballast genommen wurde. Auch bei Problemen, mit denen sich die Patienten weder an das ärztliche Personal noch ihre Angehörigen wenden wollen, sind sie für ein Gespräch mit unserer Psychologin sehr empfänglich und dankbar

Aufgrund des gravierenden Mangels an Spenderorganen können derzeit weltweit nur etwa 3000 Herztransplantationen pro Jahr durchgeführt werden. Der dauernde Mangel potenzieller Spenderorgane hat u. a. eine verschärfte Selektion der Empfänger zur Folge. Dabei erhalten psychosoziale Faktoren in der Evaluation ein besonderes Gewicht. Angesichts der hohen medikamentenbezogenen postoperativen Noncompliance - etwa $20 \%$ aller Patienten nehmen nicht, nicht regelmäßig, nicht zur vorgegebenen Zeit oder gar in exakter Dosierung ihre Immunsuppressiva - erscheint es umso wichtiger, frühzeitig psychologische und psychiatrische Kontraindikationen zu identifizieren und entsprechend zu reagieren. Daneben kann der Zuwachs an Kandidaten für eine Transplantation auf der Warteliste zu somatischen Komorbiditäten sowie einer Zunahme der psychischen Komplikationen führen. Im Klinikalltag erleben wir bereits, dass vor der Herztransplantation wochenlange, ggf. wiederholte stationäre Aufenthalte und die Konfrontation mit Komplikationen der Nährboden für psychische Belastungen in der Wartezeit sind. Auch hier sind psychologische Interventionen hilfreich.

Obwohl die Transplantationsmedizin in den vergangenen Jahren sehr erfolgreich verlaufen ist und man durchaus um die psychische Belastung der Patienten weiß, lässt im Gegensatz zur medizinischen Behandlung die psychologische 
Betreuung von Transplantationskandidaten und Transplantierten zu wünschen übrig. Selbst das im Jahr 1997 eingeführte neue Transplantationsgesetz, welches die psychologische Mitbetreuung ausdrücklich fordert, hat an dieser Situation nicht viel geändert. Festzustellen ist vor diesem Hintergrund auch, dass bisher qualitative Mindeststandards für eine psychische $\mathrm{Be}$ treuung fehlen, zu der jedes Zentrum nach $\$ 10$ Transplantationsgesetz verpflichtet ist. Dazu gehören natürlich auch die entsprechenden Fortbildungen für Ärzte und Krankenschwestern. Umso mehr ist das interdisziplinäre Konzept, dass nun auch in Oeynhausen mit der Lehrstuhlübernahme durch Prof. Gummert Einzug gehalten hat, sehr zu begrüßen. Und dieser Forderung nach entsprechender Fortbildung wird auch mit der heutigen Tagung Rechnung getragen.

\section{() Psychologische Intervention in der Herzchirurgie ist kein Luxus, sondern eine wichtige und notwendige Maßnahme.}

\section{Was ziehe ich für Konsequenzen?}

Die Notwendigkeit einer psychologischen Mitbetreuung bei Herzpatienten ergibt sich aus dem Leiden der Betroffenen. Eine rein zentriert-medizinische Sichtweise greift zu kurz, da sie vernachlässigt, dass das Ergebnis des Eingriffs nicht nur vom Ausmaß der medizinischen Resultate, sondern auch von der subjektiven psychischen Befindlichkeit der Operierten abhängt. Vergleicht man die Zahl der Patienten auf der Warteliste mit der Zahl derjenigen, die einer Transplantation zugeführt werden können, so ist leicht nachvollziehbar, wie groß der Bedarf an psychologischer Unterstützung für die $\mathrm{Pa}$ tienten ist - es ist ihnen sehr wohl bewusst, dass ein Großteil der Patienten im Verlauf der Wartezeit verstirbt. Wer also die psychische Dimension einer Transplantation vernachlässigt, gefährdet den Erfolg der gesamten medizinischen Behandlung.

Die Erfahrung hat gezeigt, dass viele Herzpatienten eine psychologische Unterstützung wünschen und benötigen. Dies gilt nicht nur wegen der grundsätzlich engen Wechselwirkung von somatischen und psychischen Prozessen im Krankheitsverlauf. Vorbehalte und Sorgen auf beiden Seiten sollen deutlich reduziert werden. Der Psychologe muss über genügend medizinische Kenntnisse verfügen, um die Patienten auch in ihren körperlichen Belangen besser verstehen zu können. Dem behandelnden Arzt dagegen muss klar sein, wie sehr sich Patienten auch einen psychologisch unterfütterten Umgang seitens der Ärzte wünschen und wie bitter dessen Fehlen oft beklagt wird. Das heißt, beide Berufsgruppen müssen lernen, genau hinzuhören. Nur durch ein kooperatives und nicht durch Konkurrenz geprägtes Arbeitsmodell kann unseren Patienten geholfen werden.

Meine sehr verehrten Damen und Herren, die Eingangsfrage war: „Psychologische Interventionen in der Herzchirurgie - Notwendigkeit oder Luxus“. Wie wird Luxus eigentlich definiert und woher kommt das Wort? Es kommt aus dem Lateinischen und bedeutet soviel wie Verschwendung. Heute versteht man unter Luxus Verhaltensweisen, Aufwendungen oder Ausstattungen, welche über das übliche Maß (den Standard) hinausgehen. Über jenes Maß, das in einer Gesellschaft als notwendig und für sinnvoll erachtet wird. Diese Definition, verbunden mit dem von mir Vorgetragenen, lässt nur eine Antwort zu: Psychologische Intervention in der Herzchirurgie ist absolut kein Luxus, sondern eine wichtige und notwendige Maßnahme.

Ich bin mir natürlich darüber im klaren, dass die hergebrachte organisatorische Grundstruktur medizinischer Einrichtungen, zu der traditionell auch die Unikliniken gehören, dem im Wege stehen mag. Aber ich bin Optimist und mir scheint eine Neuordnung, deren Struktur nicht auf traditionellen Fächergrenzen und persönlichen Befindlichkeiten basiert, sondern durch die zu bewältigenden Aufgaben definiert wird, zukunftsträchtiger und unabdingbar zu sein.

$\mathrm{Ihr}$

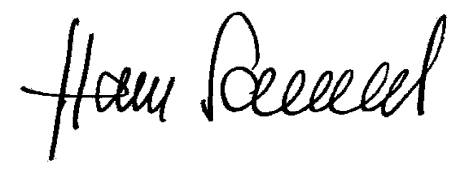

Prof. Dr. Hans H. Scheld

\section{Korrespondenzadresse}

Prof. Dr. H. H. Scheld

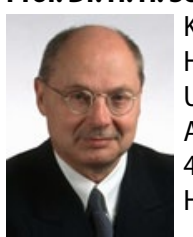

Klinik und Poliklinik für Thorax-, Herz- und Gefäßchirurgie, Universität Münster Albert-Schweitzer-Straße 33, 48149 Münster H.H.Scheld@ukmuenster.de

Prof. Dr. med. Hans H. Scheld. Jahrgang 1946. Medizinstudium an der Justus-Liebig-Universität Gießen. Weiterbildungen in den Fachrichtungen Intensivmedizin, Kardiologie, Chirurgie sowie Herz-, Gefäß- und Thoraxchirurgie. 1980 Facharzt für Chirurgie und Habilitation. 1985 Facharzt für Herz-, Thorax- und Gefäßchirurgie. 2003-2005 Präsident der Deutschen Gesellschaft für Thorax-, Herz- und Gefäßchirurgie. 1990-2011 Direktor der Klinik für und Poliklinik für Thorax-, Herz- und Gefäßchirurgie der Westfälischen Wilhelms Universität in Münster. 